\title{
A!
}

This is an electronic reprint of the original article.

This reprint may differ from the original in pagination and typographic detail.

Dhar, A.; Kinnunen, J. J.; Törmä, P.

\section{Population imbalance in the extended Fermi-Hubbard model}

Published in:

Physical Review B

DOI:

10.1103/PhysRevB.94.075116

Published: 09/08/2016

Document Version

Publisher's PDF, also known as Version of record

Please cite the original version:

Dhar, A., Kinnunen, J. J., \& Törmä, P. (2016). Population imbalance in the extended Fermi-Hubbard model.

Physical Review B, 94(7), 1-7. [075116]. https://doi.org/10.1103/PhysRevB.94.075116

This material is protected by copyright and other intellectual property rights, and duplication or sale of all or part of any of the repository collections is not permitted, except that material may be duplicated by you for your research use or educational purposes in electronic or print form. You must obtain permission for any other use. Electronic or print copies may not be offered, whether for sale or otherwise to anyone who is not an authorised user. 


\title{
Population imbalance in the extended Fermi-Hubbard model
}

\author{
A. Dhar, J. J. Kinnunen, and P. Törmä* \\ COMP Center of Excellence, Department of Applied Physics, Aalto University, AALTO, Finland
}

(Received 1 December 2015; revised manuscript received 11 July 2016; published 9 August 2016)

\begin{abstract}
We study the interplay between population imbalance in a two-component fermionic system and nearestneighbor interaction using the matrix product states method. Our analysis reveals a parameter regime for the existence of the Fulde-Ferrell-Larkin-Ovchinnikov phase. Furthermore, we find distinct evidence for the presence of hidden order in the system. We present an effective model to understand the emergent oscillations in the string correlations due to the imbalance and show how they can become an efficient tool to investigate systems with imbalance.
\end{abstract}

DOI: 10.1103/PhysRevB.94.075116

\section{INTRODUCTION}

Imbalance in the different intrinsic spin components lies at the core of systems with finite magnetic moment. It can lead to a variety of novel quantum phases such as the Fulde-FerrellLarkin-Ovchinnikov (FFLO) phase, breached pair (BP) states, fully paired and partially polarized phases, and phase separated ferromagnetic regions [1-6] in fermionic systems. On the other hand, different types of interactions can compete resulting in structure formation in nature [7,8]. Long-range interactions, in particular, induce a miscellany of phases in fermionic systems such as charge density wave (CDW), spin-density wave (SDW), bond-order wave (BOW), phase separated (PS), singlet superfluid (SSF), and triplet superfluid (TSF) phases [9-18]. In addition, it introduces a nontrivial structure in the system, one particularly being the subtle hidden order which is revealed by highly nonlocal string correlation functions. Studies on such correlation functions have revealed interesting phenomena in both bosonic and fermionic systems with long-range interactions [19-21]. But what happens when both imbalance and long-range interactions, nearest-neighbor in particular, are present in the system? Are there any indications of the presence of hidden orders in such a system? The rich interplay between these two parameters in a one-dimensional two-component fermionic system and the search for finite nontrivial string correlations are the focuses of this paper.

Recent advances in experiments using ultracold gases have opened up avenues to simulate two spin-component systems and introduce population imbalance between them [22]. The ability to trap and cool fermionic atoms with large magnetic moment has made it possible to simulate systems with long-range interactions with controllable magnitude [23-26] and image them through a quantum gas microscope [27-31] developed recently. Our results can thus be readily verified experimentally. In general, the results reveal phases of matter and increase the understanding of 1D quantum magnetism.

\section{MODEL AND METHOD}

A system of ultracold two-component fermions in a lattice with nearest-neighbor interaction can be described by the

*paivi.torma@aalto.fi extended Hubbard model (EHM) described by the Hamiltonian

$$
\hat{H}=-t \sum_{\langle i, j\rangle, \sigma} \hat{c}_{i, \sigma}^{\dagger} \hat{c}_{j, \sigma}+U \sum_{i} \hat{n}_{i \uparrow} \hat{n}_{i \downarrow}+V \sum_{\langle i, j\rangle} \hat{n}_{i} \hat{n}_{j},
$$

where $\hat{c}_{i, \sigma}^{\dagger}\left(\hat{c}_{i, \sigma}\right)$ creates (destroys) a fermion with spin $\sigma$ at site $i, \hat{n}_{i, \uparrow}=\hat{c}_{i, \uparrow}^{\dagger} \hat{c}_{i, \uparrow}\left(\hat{n}_{i, \downarrow}\right)$ is the number operator for $\uparrow(\downarrow)$ fermions at site $i$, and $\hat{n}_{i}=\hat{n}_{i, \uparrow}+\hat{n}_{i, \downarrow}$ counts the total number of particles at site $i$. The first term is due to the hopping between neighboring sites, whereas the on-site interaction between different spins is denoted by the second term. The third term takes into account the interaction between fermions on nearest neighboring sites. The above Hamiltonian at half filling has been extensively studied before, and a rich phase diagram has been reported to exist, with different correlators characterizing the respective phases [9,13-18].

In this paper, we proceed in the framework of EHM, but introduce a finite population imbalance between the two components. This is characterized by the polarization defined as $P=\frac{N_{\uparrow}-N_{\downarrow}}{N_{\uparrow}+N_{\downarrow}}$, where $N_{\uparrow}$ and $N_{\downarrow}$ are, respectively, the total number of up- and down-spin components in the system. Our primary goal is to investigate the effects of polarization on the different phases existing in the balanced scenario and the emergence of new orders in the system. In contrast, we do not intend to evaluate the entire phase diagram. Instead, we select representative points from deep inside various phases extracted from the phase diagrams of EHM in the balanced case reported earlier in the literature [9,13-16,18].

Using the matrix product states (MPS) method [32,33], we simulate a system at zero temperature of size 100 sites (unless otherwise mentioned to lift the degeneracy), with a bond dimension of 500, resulting in an error from the total weight of the discarded states to be less than $10^{-10}$. We fix the filling of spin-up particles to 50 in order to have half filling for one of the components. The half filling is a very special case in the lattice because of the symmetric single-particle lattice dispersion $\epsilon_{\pi / 2+k}=\epsilon_{\pi / 2-k}$. Since we are analyzing how the various states in the half-filled balanced situation change when spin imbalance is imposed, it is better to retain the half filling in order to avoid effects caused by the simultaneous breaking of the particle-hole symmetry in the majority component. Such essentially exact calculations of the ground state wave functions and energies are used to evaluate different correlators needed for our analysis. 


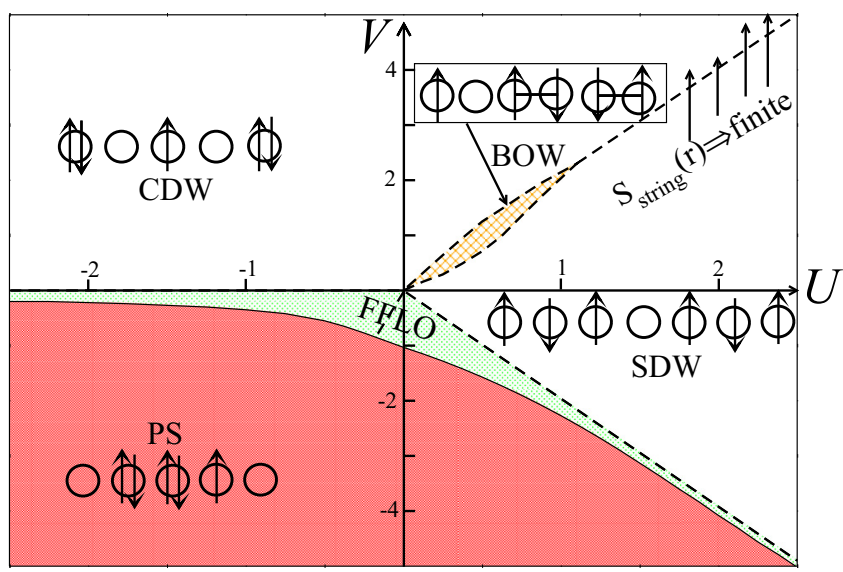

FIG. 1. Schematic representation of the different phases for the EHM being modified by the presence of imbalance. Here $U$ and $V$ are the on-site and nearest neighbor interaction energies, respectively. The diagram shows the emergence of the Fulde-FerrelLarkin-Ovchinnikov (FFLO) phase in the region occupied by singlet and triplet superfluid phases in the balanced case. Shown are also the phase separated (PS), charge-density wave (CDW), spin-density wave (SDW), and bond-order wave (BOW) phases. The appearance of a finite string correlation is discussed later in this paper. The figure is an adaptation of the corresponding phase diagram in the balanced case in Ref. [18].

\section{EFFECTS OF IMBALANCE ON DIFFERENT STATES}

We begin our analysis by looking at the charge density wave $(\mathrm{CDW})$ phase which appears in an extended region for attractive $U$, repulsive $V$, and also when both $U$ and $V$ are repulsive with $U \lesssim 2 V$, have alternate sites as doubly occupied as shown in Fig. 1. This phase is characterized by peaks in the structure factor of the density-density correlations defined as

$$
S_{\mathrm{CDW}}(k)=\sum_{r, r^{\prime}} e^{i k\left(r-r^{\prime}\right)}\left(\left\langle\hat{n}_{r} \hat{n}_{r^{\prime}}\right\rangle-\left\langle\hat{n}_{r}\right\rangle\left\langle\hat{n}_{r^{\prime}}\right\rangle\right) .
$$

For a balanced system, $N_{\uparrow}=N_{\downarrow}$, the structure factor is expected to have peaks at momentum values $k \sim \pm \pi$ in the CDW phase at half filling. Figure 2(b) indeed shows such well defined peaks of $S_{\mathrm{CDW}}(k)$. Nonzero values of the polarization $P$ decrease the value of the peaks at $k \sim \pm \pi$, indicating a decrease in the CDW character in the system as $P$ increases. This can be attributed to the decrease in the number of one of the components in the system. The decrease in the peak value is monotonic in nature as shown in Fig. 2(b).

The spin-density wave (SDW) phase is characterized by the alternate sites being occupied by up- and down-spin particles. It shows up in the structure factor of spin-spin correlations, defined as

$$
S_{\mathrm{SDW}}(k)=\sum_{r, r^{\prime}} e^{i k\left(r-r^{\prime}\right)}\left(\left\langle\hat{n}_{r}^{d} \hat{n}_{r^{\prime}}^{d}\right\rangle-\left\langle\hat{n}_{r}^{d}\right\rangle\left\langle\hat{n}_{r^{\prime}}^{d}\right\rangle\right),
$$

where $\hat{n}_{r}^{d}=\hat{n}_{r, \uparrow}-\hat{n}_{r, \downarrow}$ is the difference between spin-up and spin-down particles at a particular lattice site. In the balanced scenario at half filling, $S_{\mathrm{SDW}}(k)$ is expected to have peaks at $k \sim \pm \pi$, as observed in Fig. 2(c). With the increase in $P$, we find this peak value to decrease monotonically. In addition, a second feature is observed in the form of a small peak or

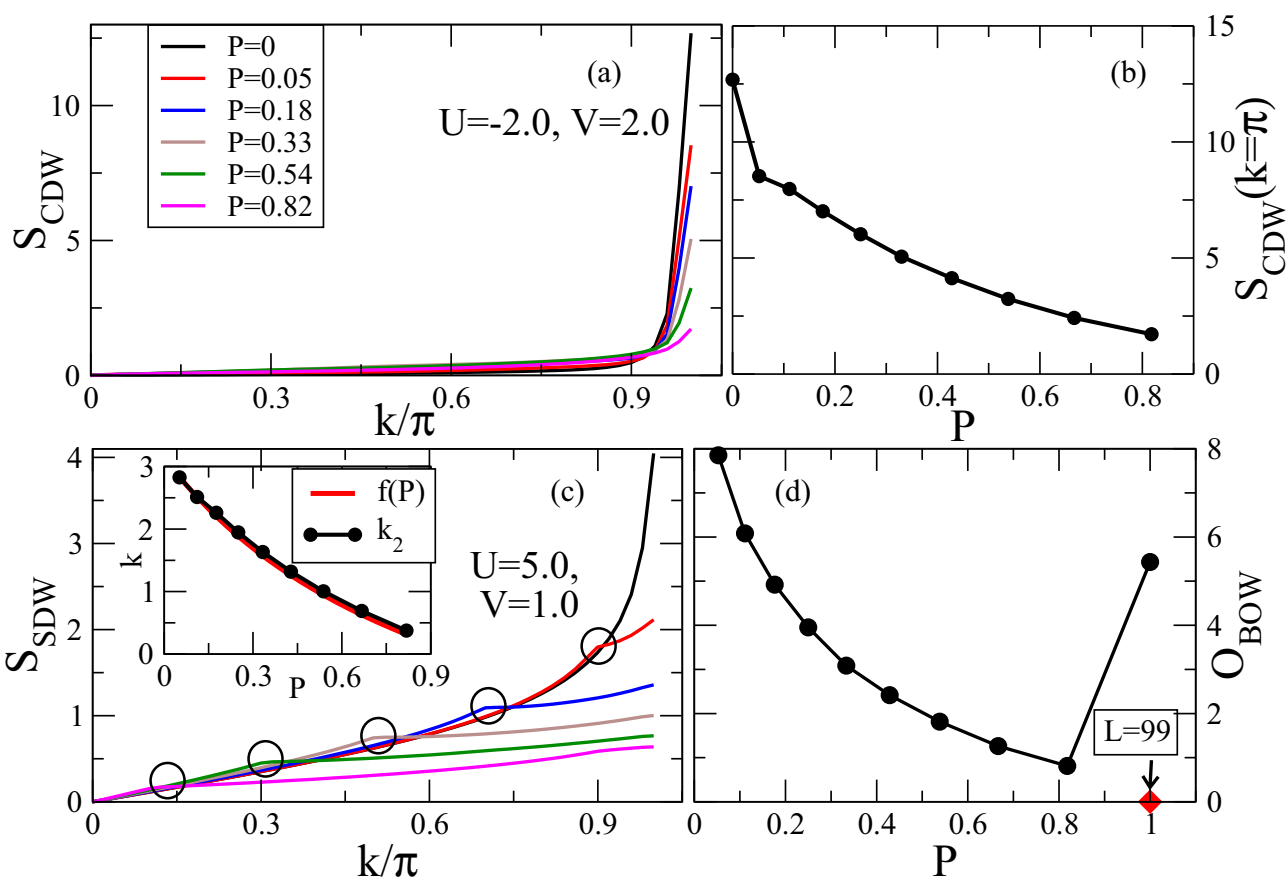

FIG. 2. (a) The density-density structure factor, $S_{\mathrm{CDW}}(k)$ for different polarizations at $U=-2.0, V=2.0$. (b) The monotonic decrease of the peak value of $S_{\mathrm{CDW}}$ at $k= \pm \pi$ shown as a function of the polarization P. (c) The spin-spin structure factor $S_{\mathrm{SDW}}(k)$ for different polarizations at $U=5.0, V=1.0$. Black circles enclose the positions of the second feature in the presence of imbalance. The color coding for the different polarizations are the same as in (a). Inset shows the momentum values corresponding to the location of the second (bump) as a function of the polarization $P$ along with the function $f(P)=\pi(1-P)\left(N_{\uparrow}+N_{\downarrow}\right) / L$. (d) The bond order wave order parameter $O_{\mathrm{BOw}}$ for different polarizations at $U=4.0, V=2.1$. The red diamond denotes the value of $O_{\mathrm{BOw}}$ when degeneracy is lifted by using 99 sites in the simulations. 


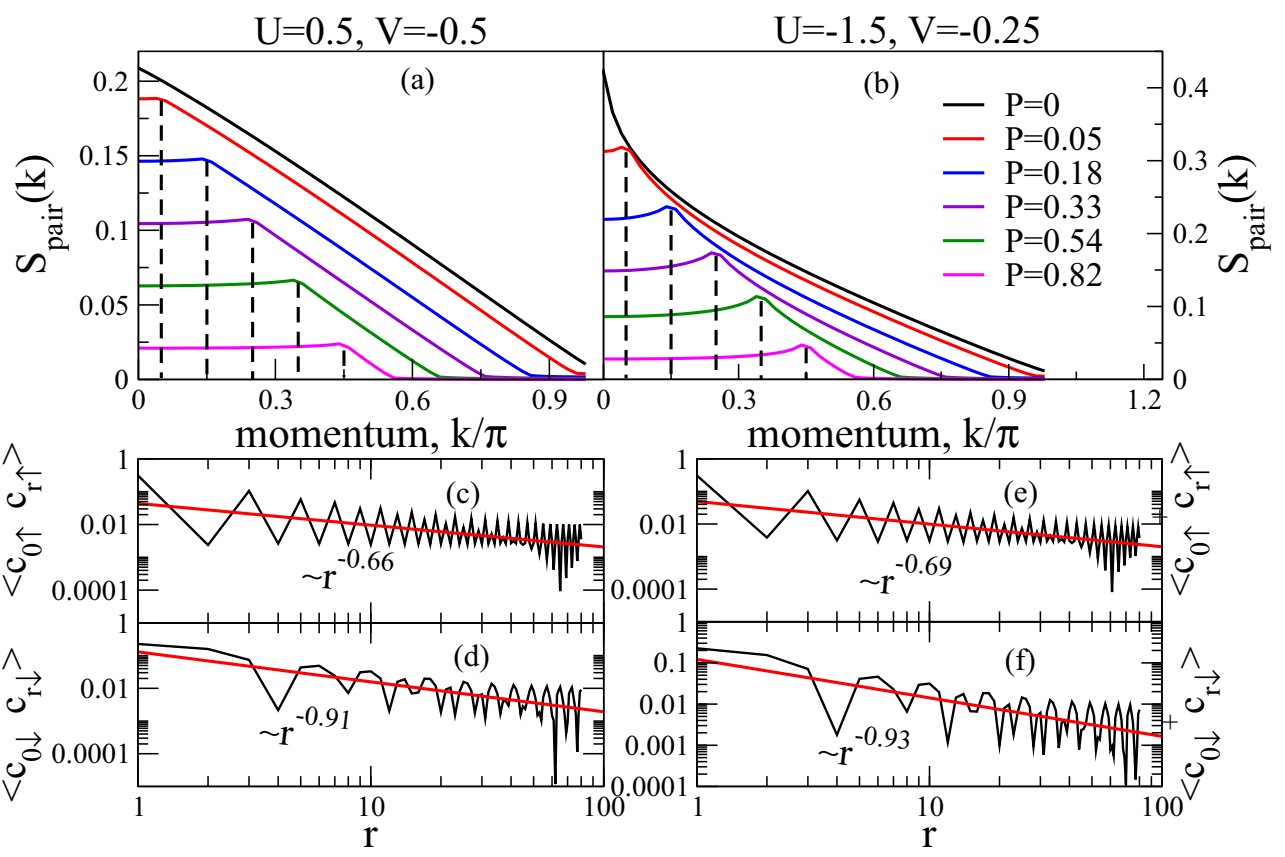

FIG. 3. (a) and (b) Plot of the pair momentum distribution, $\mathrm{S}_{\text {pair }}(\mathrm{k})$ as a function of momentum $k$ for different values of $P$ for $U=0.5$, $V=-0.5$ and $U=-1.5, V=-0.25$, respectively. The dotted lines indicate the values of the FFLO wave vector, $q$ (see text) corresponding to the different values of $P$. Single particle density matrix for spin-up [(c), (e)] and -down [(d), (f)] particles (black curves) with different values of $P$ for the same set of values of $U, V$ as in (a) and (b). The red lines indicate a power law fit to these curves with the exponent mentioned in the respective plots.

hump in $S_{\text {SDW }}(k)$, as denoted by the black circles in Fig. 2(c). This secondary feature can be attributed to the imbalance, and it indicates an additional underlying order appearing in the system. This can be understood if we consider, for example, 50 up-spin and 25 down-spin particles. On average, between every two down-spin particles, there are two up-spin particles and one empty site, resulting in a period of four sites and momentum of $\pi / 2$ for the SDW structure factor. Thus, the momentum values for these secondary features can be approximated by the function $f\left(N_{\downarrow}\right)=2 \pi N_{\downarrow} / L$, which shows excellent agreement with the actual data points as shown in the inset of Fig. 2(c).

The bond order wave (BOW) phase appears in the phase diagram of the EHM for the repulsive $U, V$ regime as shown in Fig. 1. It is characterized by BOW order parameter defined by

$$
O_{\mathrm{BOW}}=\sum_{j \sigma}\left\langle(-1)^{j}\left(\hat{c}_{j, \sigma}^{\dagger} \hat{c}_{j+1, \sigma}+\text { H.C. }\right)\right\rangle .
$$

Figure 2(d) shows the monotonic decrease of $O_{\mathrm{BOW}}$ with the increase of $P$ suggesting the BOW character to decrease with increasing imbalance. This is caused by the smaller number of down-spin particles available to bond with the up-spin particles. Interestingly, the value of $O_{\mathrm{BOW}}$ in a completely polarized case $\left(N_{\downarrow}=0\right)$ for $L=100$ increases compared to a finite $N_{\downarrow}$. This behavior can be attributed to the presence of a large number of degenerate ground states when the number of lattice sites is even. The degeneracy is lifted when we use an odd number of lattice sites $(L=99)$ leading to a very small value of the $O_{\mathrm{BOW}}$ for the fully polarized case, as indicated by the red diamond in Fig. 2(d).

We now turn our attention to the evaluation of the pair correlation function in the singlet and triplet superfluid (SSF and TSF) phases as reported earlier in the literature $[9,13$ 16,18], defined as $\rho_{i j}^{\text {pair }}=\left\langle\hat{c}_{i \uparrow}^{\dagger} \hat{c}_{i \downarrow}^{\dagger} \hat{c}_{j \downarrow} \hat{c}_{j \uparrow}\right\rangle$. The pair momentum distribution $S_{\text {pair }}(\mathrm{k})$, defined as the Fourier transform of $\rho_{i j}^{\text {pair }}$, indicates the momenta of the Cooper pairs [1,34]. In the balanced case, the pair momentum has a peak at zero momentum. Switching on the imbalance leads to peaks for nonzero values of the momentum as shown in Figs. 3(a) and 3(b). Such a behavior signals the appearance of the 1D analog of the Fulde-Ferrell-Larkin-Ovchinnikov (FFLO) phase (Refs. [1,35], and references therein). The nonzero momentum values corresponding to the peaks in $S_{\text {pair }}$ are found to be approximately equal to the FFLO wave vectors: $q \simeq$ $\pi\left(N_{\uparrow}-N_{\downarrow}\right) / L=\pi \rho P$, where $\rho=\left(N_{\uparrow}+N_{\downarrow}\right) / L$, as shown by the dotted lines in Figs. 3(a) and 3(c). We also look at the single-particle density matrix of both spin-up $\left(\left\langle\hat{c}_{0, \uparrow}^{\dagger} \hat{c}_{r, \uparrow}\right\rangle\right)$ and -down $\left(\left\langle\hat{c}_{0, \downarrow}^{\dagger} \hat{c}_{r, \downarrow}\right\rangle\right)$ particles to look for 1D superfluid (SF) signatures corresponding to these $(U, V)$ values. Figures 3(c)3(f) show the existence of long-range correlation, implying the existence of nonexponentially decaying SF order even in the presence of imbalance. Previous works have predicted the presence of the FFLO phase in the population imbalance systems with attractive $U$. In Fig. 3(a), we see signatures of FFLO phase even for repulsive $U$ with $V$ being attractive. It should be noted that due to nearest neighbor interaction, there will also be intersite pairing [17].

\section{HIDDEN ORDER}

Motivated by recent reports on the connection between hidden order and gapped phases in EHM [20,21], we look for it in the region where both $U$ and $V$ are repulsive. Earlier 

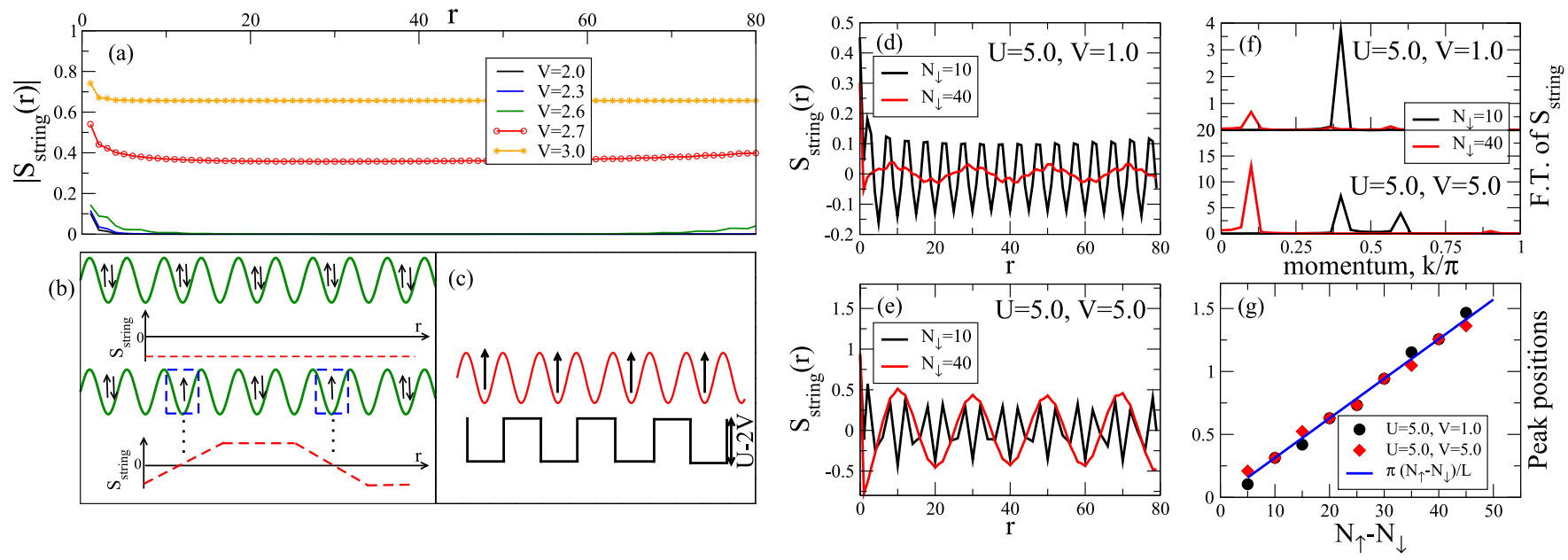

FIG. 4. (a) The absolute value of the string correlation with different values of $V$ for $U=5$ in the balanced case, showing the emergence of string order. (b) Schematic diagram to show how string correlation corresponds to the positions of the holes in the $\downarrow$ component. In the balanced case, the string correlation $S_{\text {string }}$ has a constant value of -1 shown in the top part of the figure. The bottom part shows the presence of imbalance $\left(N_{\downarrow}=N_{\uparrow} / 2\right)$, resulting in the appearance of holes as denoted by blue dashed boxes. The string correlator has nodes corresponding to the positions of the holes as indicated by the black dotted lines. (c) An effective model of a series of box potentials as seen by a single $\downarrow$ component/hole for strong interactions. (d) and (e) Plot of string correlation function for two values of polarization with $U=5, V=1$ and $U=5, V=5$, respectively. (f) The corresponding fast Fourier transforms done on the data to extract the frequencies. (g) Primary peak positions of the Fourier transforms of the string correlations as a function of spin imbalance along with the function $\pi \times\left(N_{\uparrow}-N_{\downarrow}\right) / L$.

works on the extended Bose-Hubbard model with bosons in an optical lattice $[19,36]$ had found long range string correlations characterizing the Haldane insulator phase. We define the relevant string correlation function as

$$
S_{\text {string }}\left(\left|r-r^{\prime}\right|\right)=\left\langle\delta \hat{n}_{r} e^{i \pi \sum_{k=r+1}^{r^{\prime}-1} \delta \hat{n}_{k}} \delta \hat{n}_{r^{\prime}}\right\rangle,
$$

where $\delta \hat{n}_{i}=\hat{n}_{i, \uparrow}+\hat{n}_{i, \downarrow}-1$. Figure 4(a) shows the emergence of long-range string correlations as $V$ is increased for a fixed large value of $U$. An odd number of sites $(L=99)$ is used to remove the degeneracy in the ground state. For larger values of $V$, the string correlation is seen to approach a finite value asymptotically, compared to zero for lower values of $V$, with the transition occurring close to the SDW-CDW transition at $U=2 V$. Spin imbalance creates oscillations in the string order function but, even more importantly, it induces also a finite string correlation in systems which had zero string order in the balanced scenario. We find that the origin of these effects are in the wave functions of the holes created in the $\downarrow$ component, as explained below.

To understand the connection between the origin of oscillations in the string correlation and imbalance, we consider a case for which $U<2 V$. In the homogeneous balanced scenario, the system will exhibit CDW nature as shown in the upper part of Fig. 4(b). This results in a finite string correlation function. However, removing some $\downarrow$ atoms (creating holes) in a translationally invariant system will create evenly spaced nodes in the string correlation corresponding to the locations of the holes, as indicated by the lower part of Fig. 4(b). If there are $N\left(=N_{\uparrow}-N_{\downarrow}\right)$ equally spaced nodes present in a system of $L$ sites, then the wave vector of the oscillations (or the momentum values from the Fourier transform) will be $\pi\left(N_{\uparrow}-N_{\downarrow}\right) / L$. Figure 4(f) shows the Fourier spectra of string correlations corresponding to Figs. 4(d) and 4(e) in the presence of imbalance. Figure $4(\mathrm{~g})$ shows the primary momentum values of the oscillations as a function of the imbalance, and it does show a remarkable conformity with the above scaling. The scaling $\pi\left(N_{\uparrow}-N_{\downarrow}\right) / L$ was derived here assuming localized holes as in the lower part of Fig. 4(b). However, localized holes would result in broad Fourier spectra, unlike the narrow peaks observed in Fig. 4(f).

In order to go beyond localized holes, we now present an effective single-component model. Due to nearest neighbor repulsion between the half-filled lattice of $\uparrow$ atoms, the $\uparrow$ atoms arrange themselves in a crystal order with alternating occupied/empty sites. Since the interaction is strong, the $\uparrow$ atoms are well localized, and consequently the $\downarrow$ atoms feel an effective static potential in which every odd site has an energy shift $U$ and every even site shift $2 V$. In practice, this translates into an alternating potential with wells of depth $U-2 \mathrm{~V}$ as shown in Fig. 4(c). This potential landscape results in a twoband structure in the single-particle excitation spectrum of $\downarrow$ atoms (for details, see the Appendix ). In a balanced system, in which the $\downarrow$ component is half filled, the lower band is fully filled while the upper band is completely empty.

When spin imbalance is introduced, the first created hole is in the highest lying state of the lower band. This hole has a slowly oscillating probability distribution with wavelength equal to twice the size of the system (for details, see the Appendix ). Thus the node that in the above simple picture would have been located at the center of the lattice, is now replaced by a hole that has probability distribution spread over the whole lattice, but the maximum is still at the center. This smooth spreading with the wavelength of twice the system size is intuitive as the behavior of a particle in a box [combined with the fast every-second-site modulation coming from the effective potential of Fig. 4(c)]. Increasing the spin imbalance will create more holes with faster oscillations, resulting in the Fourier spectra seen in Fig. 4(f). In addition to the dominant oscillations, the hole wave functions will also have 
contributions from states with finite probabilities of finding the particle in the effective barriers depicted in Fig. 4(c). These manifest as rapidly oscillating Fourier components, seen also as minor secondary peaks at high momenta (larger than $\pi / 2$ ) in Fig. 4(f).

The string correlator is thus sensitive to correlations between doublons and/or empty sites. Spin imbalance produces interesting effects, as it involves creating more empty sites in the SDW phase or removing doublons in the CDW state. In a homogeneous system it shares many properties with the CDW and SDW correlators. However, the phase factor $e^{i \pi \Sigma_{k} \delta \hat{n}_{k}}$ in the definition of $S_{\text {string }}$, instead of the factor $e^{i k r}$ in a Fourier transform, allows more flexibility for the correlations between doublons and/or empty sites. This makes it possible to detect orderings that would otherwise remain hidden.

\section{CONCLUSIONS}

To conclude, we have studied a system of two-component fermions in the presence of nearest-neighbor interactions described by the EHM. The introduction of spin imbalance has interesting effects on the various phases of the EHM. The CDW, SDW, and BOW phases decrease in their respective orders with the increase in polarization. An additional order appears in the SDW with the onset of imbalance. In the SSF and TSF phases, we observe formation of Cooper pairs with finite momentum. We find an extended region for which the FFLO phase persists in the presence of finite polarization. Finally, we find finite hidden order in the system. Imbalance brings in oscillations to the string correlations, even when there is no long-range string order in the balanced system. Our in-depth analysis shows that the string correlator can be a powerful tool when analyzing hole and doublon correlations in lattice systems. Our results show that the interplay of spin population imbalance and long-range interactions leads to rich physics, especially concerning exotic paired phases and hidden order.

\section{ACKNOWLEDGMENTS}

This work was supported by the Academy of Finland through its Centre of Excellence Programme (2012-2017) and under Project Nos. 263347, 251748, 284621, and 272490, and by the European Research Council (ERC-2013-AdG-340748CODE). Computing resources were provided by CSC - the Finnish IT Centre for Science and the Aalto Science-IT Project. A.D. acknowledges Michael Wall for useful discussions. The numerical simulations were carried out using the MPS open source code [37].

\section{APPENDIX A: THE EFFECTIVE MODEL FOR HOLES IN A LATTICE}

The potential landscape as shown in Fig. 4(c) of the main text results in a two-band structure in the single-particle excitation spectrum of $\downarrow$ atoms as shown in Fig. 5. The spectrum has been calculated using an exact diagonalization code. Note that the same effective model holds also for the case $U>2 V$, but then sites occupied by the $\uparrow$ atoms become barriers and empty sites become wells for the $\downarrow$ atoms. In a balanced system, in which the $\downarrow$ component is half filled, the

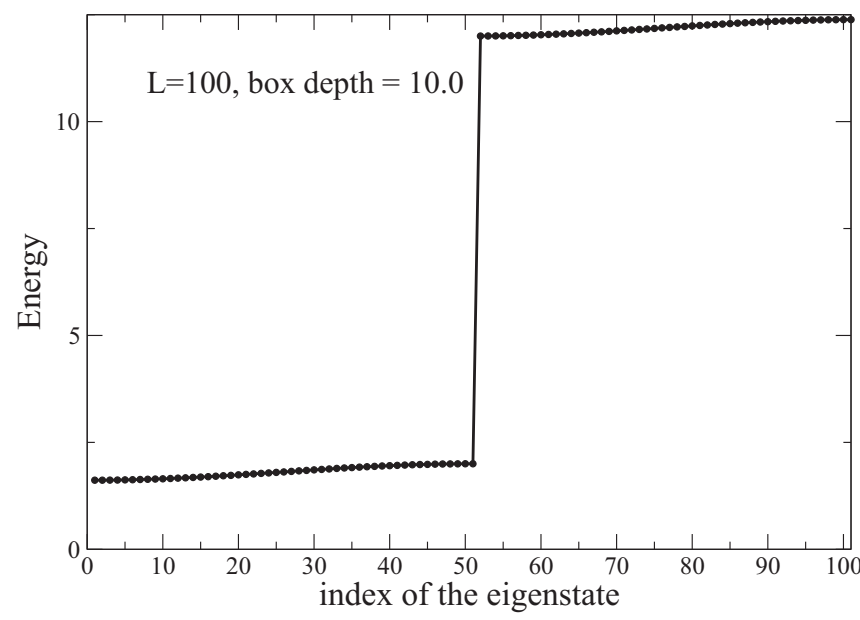

FIG. 5. The plot of the energy spectrum from the exact diagonalization code for a single particle in a series of box potentials. It clearly shows two bands with a gap between them.

lower band is fully filled while the upper band is completely empty.

When spin imbalance is introduced, the first created hole is in the highest lying state of the lower band. The wave function of this hole created will be related to the wave function of the highest state in the lower band. A closer analysis (together with the particle-hole transformation presented later) shows that this hole wave function has the same low frequency component as the ground state wave function as shown in Fig. 6. It thus explains the low frequency behavior of the string correlation oscillations for smaller number of holes compared to a larger number of holes in the system.

\section{APPENDIX B: REDUCING THE MODEL TO A SINGLE BAND}

Since the higher band of the two-band model plays no role, we will here consider a simple single-band model. This implies

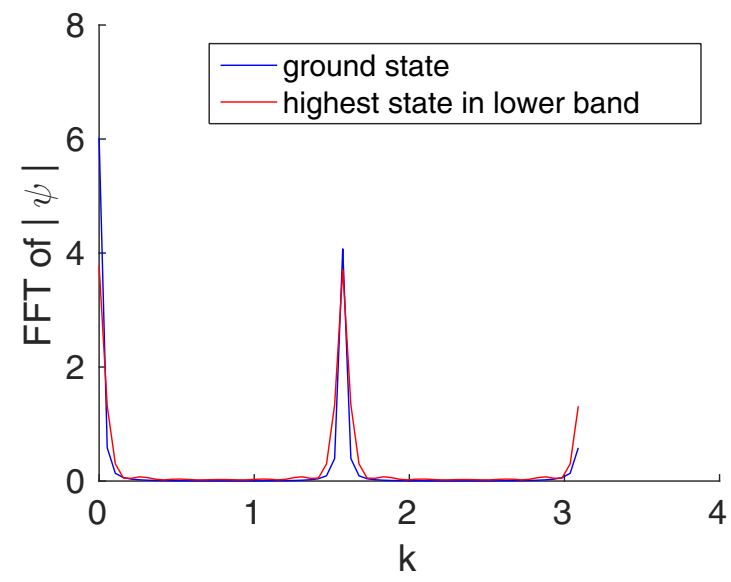

FIG. 6. Fast Fourier transform (FFT) of the absolute value of the ground state and the highest state in the lower band (which is the hole wave function) showing that they have the same frequency component. 


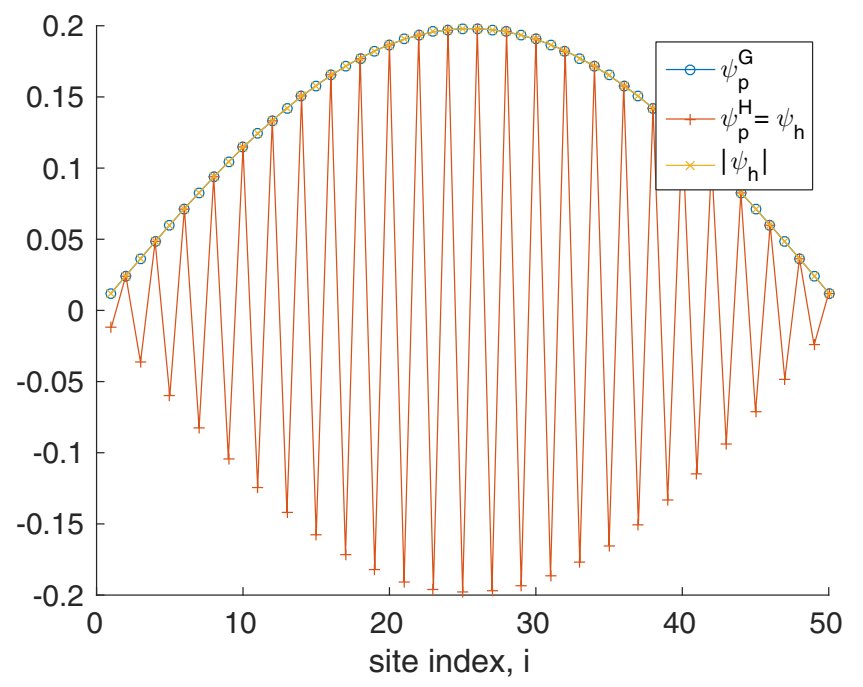

FIG. 7. Plot of the wave function corresponding to the ground state of Eq. (B1) for particles, $\psi_{p}^{G}$, and the highest state for the particles, $\psi_{p}^{H}$, which is also equal to the state of the first hole created, $\psi_{h}$. The absolute value of the hole wave function, $\left|\psi_{h}\right|$, is also plotted. State is also plotted.

a homogeneous lattice without any barriers, thus reducing our system size considered in the previous section from 100 to 50. Let us now perform the particle-hole transformation in the one-dimensional Hubbard model for noninteracting particles, given by

$$
\hat{H}=-t \sum_{\langle i, j\rangle, \sigma} \hat{c}_{i, \sigma}^{\dagger} \hat{c}_{j, \sigma}+\text { H.c. }
$$

We restrict our calculations to a single particle, hence we get rid of the spin index. We first go to the momentum basis to get the energy eigenvalues for the particle:

$$
\hat{c}_{k}=\frac{1}{\sqrt{N}} \sum_{l=1}^{N} e^{i k l} \hat{c}_{l}^{\dagger},
$$

where $N$ is the number of sites. Such a transformation will diagonalize the Hamiltonian in the momentum basis

$$
\hat{H}=-2 t \sum_{k} \cos (k) \hat{c}_{k}^{\dagger} \hat{c}_{k}=\sum_{k} \epsilon_{k} \hat{c}_{k}^{\dagger} \hat{c}_{k},
$$

where $\epsilon_{k}=-2 t \cos (k)$ denotes the dispersion relation, with the allowed momentum values given by $k_{n}=\pi n / N$, with $1 \leqslant n \leqslant N$. The energy eigenvalues are thus given by $\lambda_{n}=$ $-2 t \cos (n \pi /(N+1))$, and the eigenvectors in the position basis are given by $V_{n}=\left(\sin \frac{n \pi}{N+1}, \sin \frac{2 n \pi}{N+1}, \cdots, \sin \frac{n N \pi}{N+1}\right)$. The ground state and the highest eigenstate for the particle are shown in Fig. 7. Note that the system is finite, so instead of simply a homogeneous density for the ground state, as in an infinite lattice for $k=0$, there is a modulation with wavelength of twice the system size. For the highest state in the band, this modulation is the same but overlayed with the rapid oscillations related to the highest momentum in the band. Similar considerations would apply for hole densities in an infinite system.

We now perform the particle-hole transformation:

$$
\hat{c}_{i}=(-1)^{i} \hat{h}_{i}^{\dagger}
$$

where $h^{\dagger}$ is the creation operators for the holes. Such a transformation also divides the system into a bipartite lattice, with the odd sites getting an extra phase. This transformation will lead to a similar Hamiltonian as for the particles

$$
\hat{H}_{\text {holes }}=-t \sum_{\langle i, j\rangle, \sigma} \hat{h}_{i, \sigma}^{\dagger} \hat{h}_{j, \sigma}+\text { H.c. }
$$

with a similar energy dispersion relation, $\epsilon_{k}=-2 t \cos (k)$.

Now the hole is created by removing the particle from the highest lying state in the original problem. This is the same as adding one hole in the particle-hole transformed system, and the wave function of the hole will be similar to that of the ground state in the original problem. But because of the bipartite lattice, every alternate site will acquire an extra negative sign, resulting in that the hole wave function to be oscillating with the same large wavelength (smaller momenta) as the ground state wave function of the particle but superposed with a rapid sign changing modulation, as shown in Fig. 7. The string correlator studied in the main text involves density operators $\hat{n}_{i \sigma}=\hat{c}_{i \sigma}^{\dagger} \hat{c}_{i \sigma}$, and the correlator is thus sensitive to the density distributions. Hence the rapid phase modulations in the hole wave functions are not observed. This is precisely the reason why we observe lower momenta in the Fourier transform of the string correlator when we have a lesser number of holes.
[1] A. Korolyuk, F. Massel, and P. Törmä, Phys. Rev. Lett. 104, 236402 (2010).

[2] B. Wang, H.-D. Chen, and S. Das Sarma, Phys. Rev. A 79, 051604(R) (2009).

[3] G. Orso, Phys. Rev. Lett. 98, 070402 (2007).

[4] H. Mosadeq and R. Asgari, Phys. Rev. B 91, 085126 (2015).

[5] M. Okumura, S. Yamada, M. Machida, and H. Aoki, Phys. Rev. A 83, 031606(R) (2011).

[6] Y. Liao, A. S. C. Rittner, T. Paprotta, W. Li, G. B. Partridge, R. Hulet, S. K. Baur, and E. J. Mueller, Nature (London) 467, 567 (2010).

[7] M. Seul and D. Andelman, Science 267, 476 (1995).
[8] U. Löw, V. J. Emery, K. Fabricius, and S. A. Kivelson, Phys. Rev. Lett. 72, 1918 (1994).

[9] J. Voit, Phys. Rev. B 45, 4027 (1992).

[10] T. Giamarchi, Quantum Physics in one Dimension (Clarendon Press, Oxford, 2003).

[11] M. Nakamura, J. Phys. Soc. Jpn. 68, 3123 (1999).

[12] M. Nakamura, Phys. Rev. B 61, 16377 (2000).

[13] Y. Z. Zhang, Phys. Rev. Lett. 92, 246404 (2004).

[14] S. Ejima and S. Nishimoto, Phys. Rev. Lett. 99, 216403 (2007).

[15] M. Tsuchiizu and A. Furusaki, Phys. Rev. Lett. 88, 056402 (2002).

[16] C. Mund, Ö. Legeza, and R. M. Noack, Phys. Rev. B 79, 245130 (2009). 
[17] K. Sun, C.-K. Chiu, H.-H. Hung, and J. Wu, Phys. Rev. B 89, 104519 (2014).

[18] W. C. Yu, S.-J. Gu, and H.-Q. Lin, arXiv:1408.2642.

[19] E. G. Dalla Torre, E. Berg, and E. Altman, Phys. Rev. Lett. 97, 260401 (2006).

[20] A. Montorsi and M. Roncaglia, Phys. Rev. Lett. 109, 236404 (2012).

[21] L. Barbiero, A. Montorsi, and M. Roncaglia, Phys. Rev. B 88, 035109 (2013).

[22] P. Törmä and K. Sengstock, Quantum Gas Experiments (Imperial College Press, London, 2015).

[23] C. Trefzger, C. Menotti, B. Capogrosso-Sansone, and M. Lewenstein, J. Phys. B 44, 193001 (2011).

[24] M. A. Baranov, M. Dalmonte, G. Pupillo, and P. Zoller, Chem. Rev. 112, 5012 (2012).

[25] L. Santos, Quantum Gas Experiments (Imperial College Press, London, 2015), Chap. 13.

[26] E. A. L. Henn, J. Billy, and T. Pfau, Quantum Gas Experiments (Imperial College Press, London, 2015), Chap. 14.
[27] L. W. Cheuk, M. A. Nichols, M. Okan, T. Gersdorf, V. V. Ramasesh, W. S. Bakr, T. Lompe, and M. W. Zwierlein, Phys. Rev. Lett. 114, 193001 (2015).

[28] M. F. Parsons, F. Huber, A. Mazurenko, C. S. Chiu, W. Setiawan, K. Wooley-Brown, S. Blatt, and M. Greiner, Phys. Rev. Lett. 114, 213002 (2015).

[29] E. Haller, J. Hudson, A. Kelly, D. A. Cotta, B. Peaudecerf, G. D. Bruce, and S. Kuhr, Nat. Phys. 11, 738 (2015).

[30] G. J. A. Edge, R. Anderson, D. Jervis, D. C. McKay, R. Day, S. Trotzsky, and J. H. Thywissen, Phys. Rev. A 92, 063406 (2015).

[31] R. Yamamoto, J. Kobayashi, T. Kuno, K. Kato, and Y. Takahashi, New J. Phys. 18, 023016 (2015).

[32] U. Schollwöck, Ann. Phys. (NY) 326, 96 (2011).

[33] F. Verstraete, J. J. García-Ripoll, and J. I. Cirac, Phys. Rev. Lett. 93, 207204 (2004)

[34] C. N. Yang, Rev. Mod. Phys. 34, 694 (1962).

[35] J. Kajala, F. Massel, and P. Törmä, Phys. Rev. A 84, 041601(R) (2011).

[36] D. Rossini and R. Fazio, New J. Phys. 14, 065012 (2012).

[37] http://sourceforge.net/projects/openmps/. 